\title{
Che cosa è andato storto? Le politiche della memoria nell'epoca del post-testimone
}

\author{
di Valentina Pisanty
}

Gen 3, 2020 | In evidenza, La didattica della Shoah $|\underline{0}|$

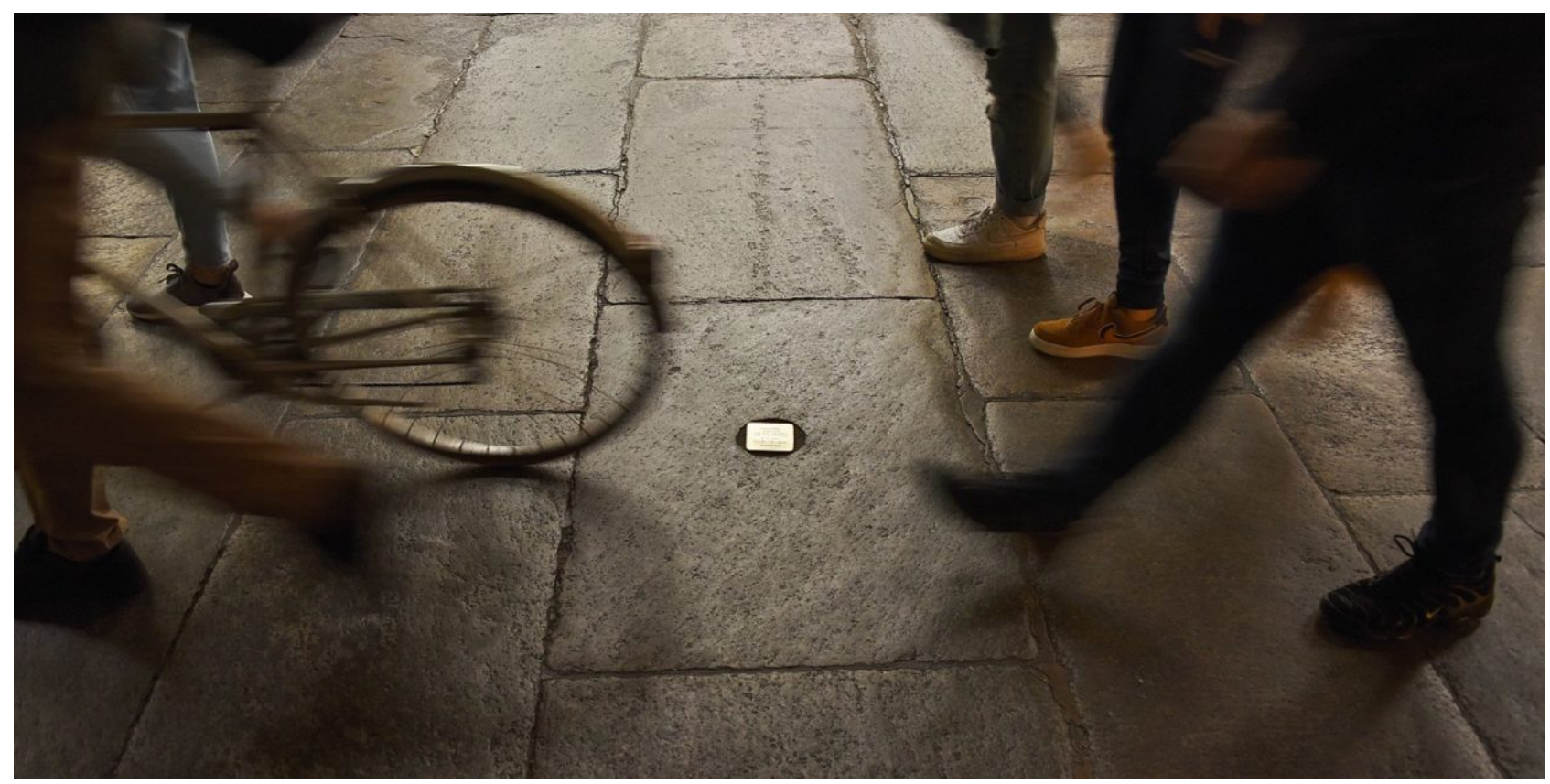

Pietra d'inciampo a Torino

Di Christian Michelides, CC BY-SA 4.0, $\underline{\text { Collegamento }}$

\begin{abstract}
Due fatti sono sotto gli occhi di tutti: 1) negli ultimi vent'anni la Shoah è stata oggetto di intense e capillari attività commemorative in tutto il mondo occidentale; 2) negli ultimi vent'anni il razzismo e l'intolleranza sono aumentati a dismisura proprio nei paesi in cui le politiche della memoria sono state implementate con maggior vigore. Sono fatti irrelati, due serie storiche indipendenti, oppure un collegamento c'è, ed è compito di una società desiderosa di contrastare l'attuale ondata xenofoba interrogarsi sulle ragioni di questa contraddizione?

La constatazione da cui trae avvio il mio intervento è il fallimento delle politiche della memoria, fondate sull'equazione semplicistica Per Non Dimenticare = Mai Più. La domanda è se tale insuccesso sia accidentale (la xenofobia cresce nonostante le politiche della memoria), o se non sia già insito nelle premesse (per come sono state impostate, quelle politiche non potevano che contribuire agli esiti che hanno prodotto). L'obiettivo è predisporsi a combattere la discriminazione in modo efficace e incisivo, che vuol dire anche onesto, consapevole e, ove necessario, autocritico.
\end{abstract}

\section{Vent'anni di attività commemorative della Shoah}

Ci sono due fatti che sono sotto gli occhi di tutti. Il primo è che negli ultimi vent'anni la Shoah è stata oggetto di capillari attività commemorative in tutto il mondo occidentale. Non mi riferisco solo al Giorno della memoria, ma anche alla vasta cinematografia sull'Olocausto, ai viaggi organizzati ad Auschwitz e ad altri luoghi del trauma, ai programmi didattici patrocinati dalle 
istituzioni,[1] ai discorsi ufficiali pronunciati dai più improbabili portavoce dell'antirazzismo in visita a Yad Vashem o all'Holocaust Memorial Museum di Washington, fino all'istituzione di leggi anti-negazioniste che, sulla falsariga della legge Gayssot del 1990, pretendono di neutralizzare chiunque neghi o minimizzi la portata storica della Shoah e di altri imprecisati crimini contro l'umanità. Da questo conglomerato di pratiche commemorative standard scaturisce un archivio di asserzioni che, a forza di essere ripetute, hanno assunto un'aria di assoluta ovvietà. Tra questi, l'idea che l'Olocausto vada inteso come l'evento spartiacque della storia del Novecento, fondativo dell'identità europea postbellica, paradigma di tutte le altre memorie «a misura di vittima»[2], pietra di paragone e schema narrativo profondo (articolato nei ruoli di Vittime, Carnefici, Bystanders e Giusti) che struttura l'immaginario occidentale, dando senso ai più disparati eventi... E contemporaneamente come un evento irrappresentabile, irripetibile, non paragonabile a nessun altro evento passato o presente, la cui memoria ciò nondimeno funge da rimedio (o barriera protettiva) contro l'insorgere di ogni nuova forma di razzismo, secondo l'equazione Per Non Dimenticare = Mai Più (Never Forget = Never Again) profondamente radicata nel senso comune di chiunque si identifichi con le narrazioni egemoni nelle odierne democrazie liberali.

\section{L'aumento di razzismo e intolleranza}

Il secondo fatto è che negli ultimi vent'anni il razzismo e l'intolleranza sono aumentati a dismisura proprio nei paesi in cui le politiche della memoria sono state implementate con maggior vigore. Episodi sempre più frequenti di violenza razzista, rivendicazioni esplicite di orgoglio nazionalistico, parate di simboli fascisti, discriminazioni sul lavoro, propagazione di odio in rete, per strada, in televisione, sui giornali e nei luoghi istituzionali, partiti xenofobi al governo e molti altri segnali preoccupanti suggeriscono che lo «scudo dell'Olocausto»[3] non stia facendo il suo lavoro. Il rapporto annuale stilato nel 2016 dalla Commissione europea contro il razzismo e l'intolleranza (Ecri) conferma l'impressione che da qualche anno a questa parte i discorsi e le pratiche razzisti stiano riconquistando il centro della vita pubblica.

Una crescente dicotomia tra «noi» e «loro» si è sviluppata nel discorso pubblico di molti paesi, con il proposito di escludere le persone sulla base del colore della pelle, della religione, della lingua o dell'etnia. Ciò non ha riguardato esclusivamente i migranti di recente arrivo, ma anche le minoranze consolidate da diverso tempo in Europa. Queste tendenze non solo minacciano un atteggiamento di accoglienza verso le persone appena giunte in Europa, ma pure il progetto più generale di costruire società inclusive e di rafforzare l'accettazione delle differenze culturali perseguito nei decenni precedenti. Sulla scia di queste tendenze, i partiti politici tradizionali, nel tentativo di prevenire un'ulteriore erosione della loro base elettorale, hanno spesso inglobato alcuni elementi di questa retorica e le idee a essa associate, amplificando così gli effetti dell'attuale ondata di populismo xenofobico e agevolando il passaggio di tali atteggiamenti dalle frange estremistiche alla politica mainstream.

Eppure la stessa commissione, per bocca dell'allora presidente Jean-Claude Juncker, il 26 gennaio del 2018 si compiace del ruolo fondamentale svolto dalle organizzazioni internazionali nella divulgazione di conoscenze sull'Olocausto per il «consolidamento delle difese contro tutte le forme di odio che minacciano le società europee» ${ }^{[4]}$. Il presupposto è che una diffusa campagna di informazione sul genocidio ebraico sia il miglior rimedio contro l'insorgenza di nuovi razzismi, fascismi, nazionalismi e inimicizie fra popoli. 
L'orrore per Auschwitz è un sentimento unificante che trascende ogni divisione culturale, economica e politica. Accendere quel sentimento fin dai primi anni di scuola, alimentarlo per mezzo di programmi di «educazione alla Shoah» $[\mathrm{sic}]^{[5]}$, rinfocolarlo di anno in anno in occasione delle feste comandate è il nostro modo per garantire la pace sociale. Così d'altronde si esprimono tutti coloro che, in contesti fortemente istituzionali, celebrano la funzione benefica della memoria con formule standard ricalcate sulla sequenza Never Forget - Never Again.

Non sto discutendo ora della prevedibilità retorica in qualche misura imposta dalla cornice celebrativa in cui vengono proferiti questi discorsi ${ }^{[6]}$. E neppure è il caso di chiedersi quanto siano sinceri i propositi che individualmente li animano, posto che in situazioni del genere è la lingua - il codice, la norma stilistica, la ritualità sociale - che parla per mezzo dei suoi ripetitori occasionali, tutto sommato fungibili. Colpisce piuttosto l'impermeabilità del sistema alle numerose smentite empiriche del suo entimema di base: la memoria della Shoah è un formidabile antidoto contro il razzismo e l'intolleranza. Possibile che - come ha recentemente osservato Henry Rousso - in un'epoca in cui «tutte le politiche pubbliche, per non dire tutte le attività quotidiane, sono sempre più soggette a forme di valutazione o di benchmarking»[7], solo le politiche della memoria siano esenti da questo genere di controllo? Perché facciamo così fatica ad ammettere che qualcosa non ha funzionato?

L'ipotesi da cui trae le mosse questa riflessione è che il fallimento delle politiche della memoria era già insito nelle sue premesse. Per come sono state impostate, quelle politiche - e le retoriche che le legittimano - non potevano che contribuire agli esiti che hanno prodotto. Per capire cosa è andato storto, occorre dissodare la retorica commemorativa, appiattita su un eterno presente, per storicizzare e problematizzare alcuni dei suoi assunti di base, a cominciare dall'entimema di base, malamente tratto da un famoso aforisma di George Santayana, secondo cui «Coloro che non ricordano il passato sono condannati a ripeterlo»[8].

\section{Mai Più}

Di solito chi proferisce gli slogan Per Non Dimenticare e Mai Più non avverte il bisogno di specificare esattamente cosa non vada dimenticato, e soprattutto cosa non debba succedere mai più. Tutti sanno che la duplice promessa riguarda l'Olocausto. Ma i termini dell'impegno restano vaghi. $\mathrm{Ci}$ si riferisce al genocidio in senso stretto (mai più stermini su scala industriale) o anche ad altre modalità di discriminazione? All'antisemitismo (mai più persecuzioni ai danni degli ebrei) o a ogni forma di razzismo e di sopraffazione? E che cosa garantirebbe il passaggio dalla prima formula (Per non dimenticare) alla seconda (Mai più)? Davvero è sufficiente ricordare ciò che è stato per tutelarsi contro l'eventualità che qualcosa di simile capiti di nuovo? Hitler ricordava perfettamente il genocidio degli armeni purtroppo.

Il concetto-cardine della Memoria contemporanea funziona come un meme, o una formula scaramantica, di cui si ignorano l'origine e il significato. Pochi ricordano il senso di chiamata alle armi attribuito allo slogan Never Again! dal suo primo divulgatore, il rabbino ortodosso Meir Kahane, controverso fondatore della Jewish Defence League e poi leader del partito dell'ultradestra israeliana Kach. Never Again! A Program for Survival si intitolava appunto un suo libro pubblicato nel 1971.

In polemica con gli ebrei americani, all'epoca più coinvolti nei movimenti per i diritti civili di altri gruppi che nella lotta per la sopravvivenza del proprio, Kahane li esortava a non farsi mai più cogliere impreparati di fronte a qualsiasi ostilità o aggressione antisemita. By any means necessary, 
avrebbe detto Malcolm X, alla cui retorica Kahane (1971) provocatoriamente si ispirava. «Per l'ebreo che è così intelligente per il bene di tutti gli altri e così ottuso quando si tratta dei propri interessi, l'amore per gli ebrei richiede un criterio politico coerente: è un bene per gli ebrei? [...] Così, e solo così, l'ebreo sopravvivrà»[9].

\section{Le aporie della memoria}

La formula universalistica intorno alla quale ruota l'attuale retorica della Memoria nasce dunque da una rivendicazione particolaristica, settaria, pensata in funzione della lotta per la sopravvivenza di un gruppo specifico. Erano d'altronde gli anni in cui lo sterminio ebraico cominciava a essere messo a fuoco come un evento a sé della storia della seconda guerra mondiale - e non come uno dei suoi diversi aspetti - grazie all'impatto mediatico del processo ad Eichmann (1961) che per la prima volta puntò i riflettori di mezzo mondo su quello che nei decenni successivi sarebbe stato riconosciuto come l'evento spartiacque della storia del XX secolo.

Come ha raccontato Hannah Arendt[10], il processo fu sapientemente pianificato dall'allora primo ministro David Ben Gurion, il quale lo considerò come un'occasione per impartire al mondo un complesso di lezioni funzionali alla difficile sopravvivenza del giovane stato israeliano: diffondere la consapevolezza sulla Shoah, pungolare i sensi di colpa delle nazioni per ottenerne il sostegno politico ed economico, costruire e rafforzare un senso di identità nazionale, convincere gli ebrei della diaspora che senza la protezione di uno Stato non avrebbero mai potuto sentirsi al sicuro nel mondo, affermare la sovranità e la potenza di Israele contro i nemici che ne invocavano la distruzione.

Nulla di riprovevole in questa grandiosa operazione di pedagogia nazionale, posto che ogni narrazione identitaria è fondata su analoghi usi strumentali della Storia, ed è normale che un grande statista quale Ben Gurion indubbiamente era sfruttasse qualsiasi risorsa, materiale o simbolica, utile al perseguimento dei suoi obiettivi strategici. Fatto sta che il nucleo centrale attorno al quale ruota l'attuale retorica universalistica della Memoria è quanto di più particolaristico si possa immaginare. Attraverso la memoria delle violenze subite, un gruppo specifico promuove il proprio programma di sopravvivenza by any means necessary. Affinché Mai più diventi un principio etico di portata universale, è necessario che l'umanità intera si faccia carico non solo della domanda «E un bene per gli ebrei? », ma anche «È un bene per qualsiasi minoranza suscettibile di discriminazione e di persecuzione?». È per l'appunto nel passaggio dal particolare all'universale che si annidano le aporie della memoria.

Non sto qui discutendo la legittimità filosofica dell'universalismo, argomento spinoso e complesso che meriterebbe una riflessione a parte. Sono convinta che Mai più - inteso in senso esteso: mai più razzismo, mai più discriminazioni nei confronti di chicchessia - sia un principio difficilmente negoziabile per cui varrebbe la pena morire. Le obiezioni che seguono riguardano invece il ricorso alla Memoria come strumento adatto a raggiungere quel fine. Perché la Memoria non può essere universale? E perché, di conseguenza, non può essere impiegata per il perseguimento di un fine universale, cioè slegato dagli obiettivi specifici di qualcuno, individuo o gruppo che sia?

Di chi è la Memoria? 
Uno dei tratti distintivi della memoria - sia individuale, sia collettiva - è il fatto di essere sempre $d i$ qualcuno che la considera come la propria emanazione e perciò ritiene di poterne fare l'uso che vuole[11]. Ecco perché, perfino nel caso in cui qualcuno rammenti male un evento a cui ha partecipato in prima persona, nessuno potrà mai spossessarlo di quel ricordo, per imperfetto che sia. Tutt'al più potrà contestare la veridicità della testimonianza (la manifestazione pubblica del ricordo privato), magari portando prove della sua inaccuratezza, ma i contenuti della rimemorazione - le tracce mnestiche di cui la testimonianza è espressione - resistono alla smentita. Questa memoria è mia (e io sono la mia memoria); può darsi che mi sbagli, so di non essere infallibile; ma, per quanto qualcun altro possa sollecitarmi a correggerne i contenuti ("guarda che ricordi male, le cose non sono andate come dici tu»), l'ultima parola spetta sempre a me («sarà anche come dici tu, ma questo è ciò che mi ricordo»).

Ora, se la piena titolarità del soggetto è inoppugnabile nel caso della memoria individuale, la faccenda si fa più complicata in rapporto alla memoria collettiva. Parlare della «nostra memoria» implica che tutti gli individui facenti capo al «noi» condividano le stesse tracce mnestiche riferite agli stessi eventi. Mettersi d'accordo sulla rappresentazione più adeguata degli eventi è di per sé un'impresa problematica (in un'intervista al Corriere della sera del 2010 Claudio Pavone definiva la memoria condivisa «un concetto privo di senso. Non c'è niente di più soggettivo della memoria: un ex partigiano e un reduce della Rsi non potranno mai avere la stessa visione del passato»)... Chi decide quali episodi vanno inclusi o omessi, quali dettagli meritano di essere enfatizzati, quali rapporti di causa ed effetto vanno istituiti, quali interpretazioni sollecitate, quali punti di vista privilegiati...? A chi spetta l'ultima parola nel caso in cui le versioni non collimino?

Far prevalere una prospettiva sulle altre significa prendere atto della natura strategica della cosiddetta memoria collettiva, strumentale agli interessi e alle sensibilità di chi in quel momento ne detiene il soft power. Si può per esempio stabilire che, in rapporto a ciò che si è voluto fare dell'Italia postbellica, la memoria partigiana aveva più motivo di essere valorizzata di quella repubblichina. È stata una decisione politica di per sé legittima - così funzionano i racconti fondativi - seppure non priva di azzardi, a cominciare dai risentimenti che presumibilmente ha suscitato in coloro che, spinti ai margini della narrativa dominante, neanche volendo avrebbero potuto identificarsi con i vissuti dei propri nemici di un tempo. Le sacche di latenza in cui si accumulano le reminiscenze neglette restano sempre a disposizione per successive messe a fuoco qualora l'egemonia dovesse passare di mano (come di fatto è successo in Italia negli anni del berlusconismo), ragion per cui, al di là dei proclami irenici, la memoria nazionale è un fattore culturale divisivo almeno quanto può essere coesivo.

\section{Il ruolo della Storia}

È questo, da Tucidide in poi, il ruolo assegnato alla Storia: istituire un luogo esterno, pubblico, sufficientemente disimplicato dai fatti da poterne ricostruire la dinamica in modo il più possibile oggettivo, sulla scorta di documenti accessibili e di criteri intersoggettivamente verificabili. Il metodo storiografico è la principale arma contro gli usi più smaccatamente strumentali della memoria. Per arginare gli usi prepotenti del passato la comunità scientifica si sottomette a un meccanismo di selezione artificiale delle ipotesi, la cui potenza argomentativa viene emancipata dagli interessi specifici di questo o quell'altro gruppo egemone (o aspirante tale).

Chi viceversa riporta le rappresentazioni del passato sul piano esclusivo della Memoria in qualche misura disconosce il carattere pubblico della Storia. Che ne sia consapevole o meno, reintroduce un principio proprietario - «l'essere-sempre-mio» dei ricordi - nella gestione dei conflitti 
rimemorativi. Il problema non è tanto stabilire come sono andate veramente le cose, e neppure in quanti modi diversi gli stessi eventi si prestano a essere raccontati, quanto rivendicare i diritti, la priorità del proprio punto di vista in rapporto a quegli eventi. C'è una prospettiva che prevale d'ufficio sulle altre. Il patrimonio da preservare è il ricordo inalienabile di un'esperienza che nessuna ricostruzione storica potrà mai svuotare dei suoi contenuti soggettivi (di qui l'insistenza sui vissuti emotivi e sensoriali dei testimoni). L'eredità da trasmettere è pertanto la narrazione unilaterale di tale esperienza, aderendo alla quale si acquisisce il diritto di auto-designarsi come «noi», co-titolari della memoria e membri del consorzio deputato alla sua gestione.

\section{Universalismo e particolarismo}

Ecco il problema. Laddove il nocciolo di ogni discorso universale è Questo vale per tutti, il punto di ogni memoria è Questo l'ho vissuto solo io. Combinati insieme, i due concetti producono uno strano ircocervo: Questo l'ho vissuto solo io e quindi vale per tutti. Ovvero: proprio perché la mia esperienza (o quella del mio gruppo) è solo mia, proprio perché sono l'unica titolare della mia (della nostra) memoria, le rivendicazioni particolari che avanzo sulla scorta di quell'esperienza e di quella memoria vanno riconosciute universalmente.

C'è un modo per trasformare questo apparente non sequitur in argomento sensato? Se il riconoscimento a cui ogni gruppo aspira si limitasse al diritto di esprimere autonomamente la propria memoria, si potrebbe fare appello a un principio universale di uguaglianza delle culture per sancire una visione pluralistica ed ecologica della semiosfera (l'ambiente culturale complessivamente inteso). Ogni comunità ha la sua memoria. Ogni memoria rappresenta una nicchia culturale, una forma di vita unica e irripetibile che come tale va preservata dall'oblio e dalla distruzione (così come nella biosfera si devono salvare le specie a rischio di estinzione). È responsabilità di tutti tutelare la diversità culturale della semiosfera e la pluralità delle memorie che la compongono.

Tuttavia negli attuali discorsi sulla Memoria la posta in gioco è più alta. Non si tratta solo di affermare che l'espressione delle diverse identità culturali è un diritto da salvaguardare collettivamente in modo che ogni gruppo ne possa usufruire in modo indipendente ed equo, chi coltivando la memoria inuit, chi quella bantu, e così via. Qui si dice che alcune memorie particolari hanno diritto di essere inglobate nella memoria universale, cioè in quel serbatoio di narrazioni cui attinge l'ipotetica comunità globale per definire le condizioni irrinunciabili del suo stare al mondo. Sulla falsariga dei criteri adottati dal Registro della Memoria del Mondo patrocinato dall'Unesco [unesco.it/it/ItaliaNellUnesco/Detail/188], a queste memorie scelte si riconosce un «significato universale eccezionale». Come la dieta mediterranea, il canto polifonico georgiano, le danze della Bretagna e l'arte tessile del Perù, vengono annoverate tra i patrimoni immateriali dell'umanità da proteggere contro «l'amnesia collettiva, la negligenza e la distruzione intenzionale e deliberata».

Nell'argomento ecologico si insinua un principio di prestazione e di selezione del più adatto. Posto che la vita delle culture è fatta di - ed è resa possibile da - una serie incessante di perdite, amnesie, negligenze e distruzioni deliberate, pena il sovraccarico di informazioni che impedisce a nuove forme di vita di prendere piede in un ambiente saturo di vestigia del passato (si veda l'argomento di Nietzsche sui rischi della memoria ipertrofica), che cosa fa sì che alcune memorie particolari siano ritenute più meritevoli di altre di sottrarsi ai meccanismi fisiologici della dimenticanza? Che cosa, in altre parole, dovrebbe indurre la comunità mondiale a intervenire di peso per contrastare, ma solo in alcuni casi, l'azione erosiva del tempo? 


\section{Il caso Holocaust}

Proseguiamo con il nostro racconto. Eravamo rimasti al punto in cui, dopo il processo Eichmann (e sempre di più dopo la Guerra dei Sei Giorni, 1967, e la Guerra dello Yom Kippur, 1973), la comunità ebraica americana cominciava a preoccuparsi delle sorti di Israele e, contestualmente, a valorizzare la memoria dell'Olocausto come patrimonio identitario da tutelare e da spendere sulla scena pubblica. Erano gli anni in cui Elie Wiesel rivendicava il genocidio come «un capitolo glorioso della nostra storia eterna»[12], Meir Kahane lanciava il grido Never Again! e i portavoce ufficiali dell'ebraismo statunitense si decidevano a potenziare lo «scudo dell'Olocausto».

$\mathrm{Fu}$ in quel contesto che, nell'aprile del 1978, la Nbc mandò in onda la miniserie TV Holocaust. Il lancio fu accompagnato da una massiccia campagna pubblicitaria coordinata dalla $\mathrm{Nbc}$ insieme all'Anti-Defamation League e all'American Jewish Comittee. L'impatto fu enorme - circa cento milioni di spettatori in prime time - e si dice che «agli americani siano state impartite più informazioni sull'Olocausto in quelle quattro serate che in tutto il trentennio precedente».

Perché proprio allora? Perché un grande network statunitense, determinato a ottenere il massimo profitto dai propri investimenti, nel 1978 puntò sulla storia di una famiglia ebraica tedesca perseguitata, deportata e falcidiata dai nazisti in Ucraina e in Polonia? Le motivazioni che indussero la $\mathrm{Nbc}$ a investire sullo sceneggiato erano di ordine commerciale: si trattava di rispondere al successo incassato l'anno prima dalla Abc con Roots, sempre di Marvin Chomsky. E difatti l'impianto narrativo di Holocaust richiamava quello già collaudato dal network rivale con Roots: la narrativizzazione in forma di saga familiare di uno dei più abominevoli crimini storici subiti da un gruppo etnico «razzistizzato». L'intento pedagogico di Holocaust - sensibilizzare gli americani, e il mondo intero, su un trauma storico che il caso Eichmann aveva riattualizzato, ma che ancora non aveva proiettato nel cuore della cosiddetta Memoria Collettiva - era senz'altro al centro della campagna di lancio del prodotto televisivo. Ma a farsene carico furono principalmente le organizzazioni ebraiche alle quali la historical fiction fornì un canale di divulgazione straordinariamente efficace.

\section{Una memoria per l'America}

Resta da capire perché gli spettatori americani aderirono con tale slancio all'iniziativa della Nbc. Appassionandosi alla storia della famiglia Weiss, si proiettarono nella memoria del genocidio ebraico (incorporata nella grande narrazione americana) a dispetto - o forse in virtù - della distanza geografica, temporale, politica e culturale che li separava dal trauma in questione. L'ipotesi di alcuni storici è che, in uno scenario (post-Vietnam e post-Watergate) di sbando politico e di crisi morale, la visione di Holocaust costituì per gli americani una sorta di lavacro, un rituale pubblico tramite il quale riaffermare i valori della nazione in antitesi rispetto al «male assoluto» che fu il nazismo.

I critici più entusiasti elogiarono l'efficacia con cui Holocaust induceva il pubblico a immedesimarsi non solo con i membri della borghesissima famiglia Weiss, ma fino a un certo punto anche con gli stessi ufficiali nazisti, i quali non venivano raffigurati come una manica di sadici assetati di sangue, bensì come mediocri arrivisti smaniosi di assecondare le richieste dei capi. La questione etica era esposta con didascalica chiarezza: come vi sareste comportati voi, cari telespettatori, se vi foste trovati in circostanze analoghe? Avreste avuto il coraggio di opporvi? 
Se ne ricavava una lezione universale sul valore della responsabilità individuale, come da narrazione fondativa americana. La stessa lezione che avrebbe ispirato il progetto dell'Holocaust Memorial Museum annunciato dall'amministrazione Carter sei mesi dopo la messa in onda di Holocaust (la tempistica non è casuale: Carter doveva farsi perdonare dalla comunità ebraica il supporto alla causa palestinese e la vendita di 60 caccia F-15 all'Arabia Saudita), salvo generare infinite discussioni su quali altre minoranze perseguitate avessero diritto di rappresentanza all'interno del Museo (il dibattito sul genocidio armeno fece sfiorare l'incidente diplomatico con la Turchia).

Va da sé che una simile «americanizzazione della memoria» non poteva che comportare una ipersemplificazione spettacolare della storia dello sterminio, appiattita su schemi narrativi ipercollaudati come si conviene a qualsiasi prodotto ben confezionato dell'industria culturale. Mentre arrotondava gli spigoli della storia, la miniserie trasformava l'Olocausto in artefatto culturale facile da afferrare, pratico da maneggiare e pertanto accessibile a chiunque avesse dimestichezza con i valori proclamati dalle democrazie occidentali, da un lato, e con le regole dello show business, dall'altro. Ma la banalizzazione era il prezzo da pagare affinché la memoria del trauma trascendesse i confini ristretti del gruppo vittimizzato e si propagasse verso il centro dell'immaginario collettivo, stilizzandosi man mano che i ricordi pulsanti dei testimoni si cristallizzavano in motivi narrativi e categorie concettuali ad ampio spettro, in grado di addensare attorno a sé il consenso di una comunità allargata.

\section{Gli usi pubblici nazionali della memoria dell'Olocausto}

Le polemiche non tardarono a manifestarsi. Il giorno stesso della prima messa in onda il New York Times pubblicò una stroncatura di Elie Wiesel in cui l'autore accusava la miniserie di raffigurare «ciò che non può e che non deve essere rappresentato»[13]. Analoghe proteste si registrarono in Francia nei mesi immediatamente successivi, quando diversi critici e personaggi politici si opposero all'acquisto dei diritti di Holocaust con la motivazione che gli americani si erano indebitamente appropriati di un trauma storico non loro (alla fine lo sceneggiato fu mandato in onda da Antenne 2 con il benestare di Simone Veil, ex-deportata ad Auschwitz).

Nella Repubblica federale tedesca invece Holocaust stimolò un intenso dibattito nazionale sulle colpe della Germania e le responsabilità dei suoi cittadini, catalizzando un processo virtuoso già avviato nel corso degli anni Cinquanta e Sessanta, quando le generazioni nate durante e dopo la guerra cominciarono a chiedere conto alle più anziane della loro partecipazione ai crimini nazifascisti (gli italiani curiosamente riuscirono a tenersene fuori, recependo lo sceneggiato americano con il distacco di chi aveva la coscienza a posto)[14].

Fermiamoci qui, prima di concludere. Con l'eccezione della Germania, unico caso noto - per forza, si dirà - di appropriazione autocritica della Memoria, tutti gli altri usi pubblici dell'Olocausto rispondono alle esigenze delle proprie autonarrazioni specifiche: celebrare la nazione risorta dalle ceneri (Israele), chiamarsi fuori dalle fasi peggiori dello sterminio (Italia e Francia), esaltare il proprio ruolo di liberatori (Usa e Gran Bretagna), insistere sulla rievocazione dei crimini nazisti per schermare il ricordo di altri crimini nei quali si è più colpevolmente implicati (Usa).

In ogni caso la memoria ritorna sui binari dell'autoassoluzione, delle amnesie opportunistiche e della celebrazione di sé. Lungi dallo svolgere una funzione al contempo critica, progettuale e coesiva (le condizioni indispensabili affinché dal Never Forget si passi al Never Again), la commemorazione àncora ciascuna comunità particolare ai suoi miti fondativi, perpetuandone le 
distorsioni, soffocandone (ma non superandone) le divisioni, e ammantando l'inevitabile parzialità del racconto egemone.

\section{Una Memoria per l'Europa?}

Un'ultima osservazione sulla cosiddetta identità europea. Oggi siamo abituati a considerare il ricordo dello sterminio come la pietra miliare della coscienza europea. Così abituati che rimaniamo stupiti quando qualcuno ci rammenta che l'«europeizzazione dell'Olocausto» è in effetti una questione piuttosto recente[15]. Il processo ebbe inizio negli anni Novanta, poco dopo la caduta del Muro di Berlino, man mano che i paesi ex-comunisti entrarono nella sfera di influenza della Nato e fecero domanda per essere ammessi in Europa. Sino ad allora poco propensi a commemorare il genocidio ebraico - che pure nelle sue fasi più acute si era consumato proprio nei territori occupati dai nazisti nell'Europa dell'Est - gli stati che aspiravano al riconoscimento occidentale furono posti di fronte a un duplice compito. Da una parte sviluppare in fretta e furia la propria memoria specifica dell'Olocausto, in alcuni casi molto difficile da innestare nella narrativa nazionale, date le censure e le rimozioni che nei decenni precedenti avevano steso un velo sugli episodi di antisemitismo autoctono (si pensi ai pogrom e ai saccheggi dei beni ebraici in Polonia e in altri paesi dell'est Europa), mentre i ricordi traumatici delle persecuzioni subite per mano dei nazisti prima, e dei sovietici poi, avevano da essere a loro volta disseppelliti ed elaborati pubblicamente. Dall'altra parte si trattava di aderire ai formati della memoria europea (in buona parte filtrata attraverso i prodotti dell'industria culturale americana), tutta incentrata sulla sofferenza degli ebrei, sulla malvagità dei nazisti e sulla miseria morale dei bystanders, cioè di coloro che assistettero ai massacri senza intervenire in favore delle vittime, o addirittura collaborarono con i carnefici per il proprio sordido tornaconto. Come rappresentarsi vittime dei crimini nazisti e dell'oppressione sovietica (quest'ultima essendo la ferita più fresca e dolente), e al contempo non solo riconoscere il primato della sofferenza ebraica sulla propria, ma anche ammettere di avervi in qualche misura contribuito?

Chiunque si può identificare con le vittime del Male assoluto. Ma è appunto questo il problema: le aporie della «memoria cosmopolita» si annidano nel contrasto tra la presunta universalità del racconto-matrice e l'inevitabile particolarità degli usi che se ne fanno. Adattata a una vasta gamma di contesti storici, la narrazione dello sterminio ha modellato l'immaginario politico degli ultimi trent'anni, riconducendo ogni conflitto allo schema vittime-carnefici (talvolta con catastrofiche retroazioni, come nel caso delle guerre nella ex Iugoslavia)[16]. Di qui la concorrenza delle vittime e le accuse di lesa memoria che ciascun gruppo lancia ai gruppi rivali.

\section{Una memoria transnazionale niente affatto pacifica}

Nei paesi più attraversati da simili contraddizioni - Polonia, Ucraina, Lituania, Ungheria, Romania, Moldavia... - la messa a punto di memorie nazionali compatibili con i requisiti europei ha incontrato parecchie resistenze. Lo si evince, tra l'altro: dalle polemiche scoppiate in Polonia nel 2012 in occasione dell'uscita di Pokłosie (Aftermath), film liberamente ispirato al pogrom di Jedwabne; dall'accusa di lesa nazione rivolta dal governo di Andrzej Duda allo storico Jan Tomasz Gross nel 2016 dopo che in un articolo per Die Welt aveva scritto che «i polacchi, giustamente orgogliosi della loro resistenza anti-nazista, in effetti uccisero più ebrei che tedeschi durante la guerra»[17]; dalla Legge sull'Olocausto approvata dal parlamento polacco nel gennaio del 2018 per fissare una pena massima di tre anni di carcere per chiunque, polacco o straniero, accusi la Polonia di complicità con i crimini nazisti o si riferisca ai campi di sterminio nazisti definendoli polacchi; e 
dalla sempre più martellante campagna di odio fomentata dagli ultranazionalisti filogovernativi contro la direzione del museo di Auschwitz, rea di promuovere le «narrative straniere» ai danni della reputazione del paese...

Questi sussulti di sciovinismo xenofobo sono forse il prezzo del «biglietto di ingresso per l'Europa» acquistato dalla Polonia nel 2004, quando l'allora presidente Aleksander Kwaśniewski riconobbe le sofferenze subite durante la guerra dagli ebrei polacchi, comprese quelle inferte dai connazionali? Oppure si sarebbero manifestati comunque, e semmai è merito delle istituzioni intergovernative di avere scoperchiato il vaso e sostenuto quella parte della cittadinanza che viceversa era pronta a voltare pagina? Non c'è bisogno di deciderlo, entrambe le spiegazioni potrebbero essere vere. Fatto sta che la fusione di memorie locali e globali preconizzata dagli strateghi del cosmopolitismo europeo è stata ed è molto meno pacifica del previsto. A posteriori è legittimo chiedersi quanto fosse realistica l'idea di una memoria transnazionale interamente fondata sull'autocritica.

\section{Bibliografia}

- H. Arendt, Eichmann in Jerusalem. A Report on the Banality of Evil, Penguin, London 1963.

- A. Assmann, Transformations of Holocaust Memory, in O. Kobrynskyy, G. Bayer (a cura di), Holocaust Cinema in the Twenty-First Century: Memory, Images, and the Ethics of Representation, Wallflower, New York 2015

- M. Berenbaum, The Specter of Anti-Semitism, in "Washington Post", 1 dicembre 1982, citato in P. Novick, The Holocaust in American Life, Houghton-Mifflin, Boston 1999, p. 177

- L. David, Holocaust Discourse as a Screen Memory: the Serbian Case, in S. Jovanović, V. Stančetić (a cura di), History and Politics in the Western Balcans: Changes at the Turn of the Millenium, Center for Good Governance Studies, Belgrad 2013

- D. Giglioli, Critica della vittima, Nottetempo, Milano 2014

- J.T. Gross, Neighbors. The Destruction of the Jewish Community in Jedwabne, Princeton University Press, Princeton 2001 (trad. it. di Luca Vanni, I carnefici della porta accanto, Mondadori, Milano 2002)

- J.T. Gross, Die Osteuropäer haben kein Schamgefühl, in "Die Welt", 13 settembre 2015.

- M. Kahane, Never Again! A Program for Survival, Nash Publishing Corporation, New York 1971

- M. Kucia, The Europeanization of Holocaust Memory in Eastern Europe, in "East European Politics and Societies and Cultures", vol. 30, n. 1, SAGE 2015

- D. Levi, N. Sznaider, Memory Unbound. The Holocaust and the Formation of a Cosmopolitan Memory, in "European Journal of Social Theory", 5 (1), SAGE 2002

- B. Molden, Mnemonic Hegemony? The Power Relations of Contemporary European Memory, in J. W. Boyer, B. Molden (a cura di), eutropes: the Paradox of European Empire, University of Chicago Press, Chicago 2014

- B. Molden, Resistant Pasts vs. Mnemonic Hegemony: on the Power Relations of Collective Memory, in "Memory Studies", 9/2, 2016

- F. Nietzsche, Sull'utilità e il danno della storia per la vita, Adelphi, Milano 1974, trad. it. di G. Sossio (ed. or. 1874)

- P. Novick, The Holocaust in American Life, Houghton-Mifflin, Boston 1999

- C. Pavone, Tra i partigiani e i fascisti non può esistere "memoria comune", in "Corriere della sera", 26 novembre 2010 
- V. Pisanty, I Guardiani della Memoria e il ritorno delle destre xenofobe, Bompiani, Milano 2020

- P. Ricoeur, La memoria dopo la storia, conferenza tenuta a Roma, giugno 2001: filosofia.it/archivio/images/download/argomenti/Ricoeur_Memoria_dopo_la_storia.pdf,

- P. Ricoeur, La memoria, la storia, l'oblio, Cortina, Milano 2003, p. 169 (ed. or. P. Ricoeur, La mémoire, l'histoire, l'oubli, Seuil, Paris 2000, trad. it. di D. Iannotta)

- H. Rousso, Face au passé, Belin, Paris 2016

- G.E. Rusconi, Germania: un passato che non passa. I crimini nazisti e l'identità tedesca, Einaudi, Torino 1987

- G. Santayana, The Life of Reason: Reason in Common Sense, Scribner's, New York 1905

- I. Wallerstein, European Universalism. The Rhetoric of Power, New Press, New York 2006

- E. Wiesel, Trivializing the Holocaust: Semi-Fact and Semi-Fiction, in "New York Times", 16 aprile 1978

- A. Wieviorka, L'era del testimone, Cortina, Milano 1999 (ed. or. A. Wieviorka , L'Ère du témoin, Plon, Paris 1988, trad. it. di F. Sossi)

Note:

[1] Ogni anno il Progetto «mappatura» dell'insegnamento della Shoah negli Atenei italiani chiede ai dipartimenti l'elenco delle iniziative promosse per diffondere la cultura della memoria in funzione di non so più quale quota premiale, dove si vede come il ricordo pubblico della Shoah si innesti sulla cosiddetta cultura del merito che oggi ha invaso l'accademia e non solo.

[2] Si veda D. Giglioli, Critica della vittima, Nottetempo, Milano 2014.

[3] L'espressione è di Michael Berenbaum, 1982, cit. in P. Novick, The Holocaust in American Life, Houghton-Mifflin, Boston 1999, p. 177.

[4] ec.europa.eu/italy/news/20180126_messaggio_di_juncker_giorno_della_memoria_olocausto_it

[5] edscuola.eu/wordpress/?p=99310

[6] Seppure non manchino esempi contrari, a riprova che un'altra struttura argomentativa è possibile: il discorso di Sergio Mattarella del 25 gennaio 2018, che esprimeva il timore che la memoria autoassolutoria del fascismo italiano generi nuovi mostri, deviava significativamente dalla norma.

[7] H. Rousso, Face au passé, Belin, Paris 2016, p. 24.

[8] G. Santayana, The Life of Reason: Reason in Common Sense, Scribner's, New York 1905, p. 284.

[9] M. Kahane, Never Again! A Program for Survival, Nash Publishing Corporation, New York 1971, pp. 236-237.

[10] H. Arendt, Eichmann in Jerusalem. A Report on the Banality of Evil, Penguin, London 1963. 
[11] Sull'aspetto irriducibilmente proprietario della memoria (in quanto contrapposta alla storia), cfr. P. Ricoeur, La memoria dopo la storia, conferenza tenuta a Roma, giugno 2001:

filosofia.it/archivio/images/download/argomenti/Ricoeur_Memoria_dopo_la_storia.pdf, e P. Ricoeur, La memoria, la storia, l'oblio, Cortina, Milano 2003 (ed. or. P. Ricoeur, La mémoire, l'histoire, l'oubli, Seuil, Paris 2000, trad. it. di D. Iannotta).

[12] E. Wiesel, discorso tenuto al simposio sui "Valori ebraici dopo Auschwitz" patrocinato dalla rivista Judaism, New York, 26.3.1967, cit. in Chaumont, La concurrence des victimes, La Découverte, 2002, p. 113

[13] E. Wiesel, Trivializing the Holocaust: Semi-Fact and Semi-Fiction, in "New York Times", 16 aprile 1978.

[14] Cfr. G.E Rusconi, Germania: un passato che non passa. I crimini nazisti e l'identità tedesca, Einaudi, Torino 1978.

[15] Cfr. D. Levi, N. Sznaider, Memory Unbound. The Holocaust and the Formation of a Cosmopolitan Memory, in "European Journal of Social Theory", 5(1), sage 2002; M. Kucia, The Europeanization of Holocaust Memory in Eastern Europe, in "East European Politics and Societies and Cultures", vol. 30, n. 1, sage 2015; B. Molden, Mnemonic Hegemony? The Power Relations of Contemporary European Memory, in J.W. Boyer, B. Molden (a cura di), eutropes: the Paradox of European Empire, University of Chicago Press, Chicago 2014 e Idem, Resistant Pasts vs.

Mnemonic Hegemony: on the Power Relations of Collective Memory, in "Memory Studies", 9/2, 2016.

[16] Cfr. L. David, Holocaust Discourse as a Screen Memory: the Serbian Case, in S. Jovanović, V. Stančetic (a cura di), History and Politics in the Western Balcans: Changes at the Turn of the Millenium, Center for Good Governance Studies, Belgrad 2013.

[17] J. T. Gross, Die Osteuropäer haben kein Schamgefühl, in "Die Welt", 13 settembre 2015. 